\title{
On a Non Logsymplectic Logarithmic Poisson Structure with Poisson Cohomology Isomorphic to the Associated Logarithmic Poisson Cohomology
}

\author{
Joseph Dongho ${ }^{1}$ \\ ${ }^{1}$ Department of Mathematics and Computer Science, University of Maroua, Cameroon \\ Correspondence: Joseph Dongho, Department of Mathematics and Computer Science, University of Maroua, Cameroon. \\ E-mail: josephdongho@yahoo.fr
}

Received: November 26, 2016 Accepted: December 28, 2016 Online Published: January 23, 2017

doi:10.5539/jmr.v9n1p109 URL: http://dx.doi.org/10.5539/jmr.v9n1p109

\begin{abstract}
The main purpose of this article is to show that there are non logsymplectic Poisson structures whose Poisson cohomology groups are isomorphic to corresponding logarithmic Poisson cohomology groups.
\end{abstract}

Keywords: log-symplectic structure, poisson cohomology, log-poisson cohomology, poisson structures

\section{Introduction}

Symplectic geometry was discoved in 1780 by Joseph Louis Lagrange when he considered the non constants variable and defined the bracket of two such elements. From symplectic manifold, Poisson defined his brackets as tool for classical dynamics. Charles Gustave Jacobi realized the importance of those bracket and elucidated their algebraic properties. Sophus Lie and others authors began the study of their geometry. Connection of poisson geometry with numbers of areas including harmonic analytic, mechanics of particles and continua; completely integrable systems, justify this recent development. It is interested to recall that number of proprieties and results in this theory was developed in the case of differential manifold. Too few authors have worked in the case of singular varieties. J. Huebschmann in (Huebschmann, J., 1990) study in 1990 Poisson algebra and apply its Lie-Rinehart cohomology in the study of their geometric quantization. A. Polishchuk in (Pichereau, A., 2006) study in 1997 the Poisson brackets in algebraic framework.

In 2002 Ryushi Goto (Goto, R., 2002), with the aim of generalizing the approach of the symplectic, Atiyah class to the construction of the invariants of knots, defined the logsymplectic manifold and study several examples. The notion of logsymplectic manifold is based on the theory of logarithmic differential forms extensively study in (Saito, K., 1980). Logsymplectic manifold is simply a complex manifold $X$ equipped with a symplectic form $\omega$ that has simple poles along a hypersurface $D \subset X$. In other words, Poisson structures defined on $X-D$ by any logsymplectic form $\omega$ extends to a Poisson bracket on all $X$ whose pfaffian in a reduced defining equation for $D$. Logsymplectic manifolds can arise when one attempts to compactify symplectic manifolds. Many modulis space in algebraic geometry and gauge theory come equipped with logsymplectic structure. Such Poisson structure can then play an important role in geometric quantization of many classical observable. According to I. Vaisman in (Vaisman, I., 1991), obstruction of quantization of such classical space is measure by Poisson cohomology. But it style very difficult to determine explicit form of Poisson cohomology as we can see in (Pichereau, A., 2006) and (Monnier, Ph., 2002).

In other to propose an alternative method in the computation of such Poisson cohomology, the first author introduce in (Dongho, J., 2012) the notion of logarithmic principal Poisson structure and prove that such Poisson structure induced a Lie-Rinehart structure on the module of logarithmic differential form along a finite generated ideal $I$, from which he introduce the notion of logarithmic Poisson cohomology, and prove that such logarithmic Poisson cohomology are in general different to the associated Poisson cohomology. It was also prove in (Dongho, J., 2012) that when logarithmic Poisson structure are logsymplectic one, the two, Poisson cohomology and logarithmic Poisson cohomology are equivalent. The main objectif of this paper is to prove that there are non logsymplectic Poisson structure with isomorphic Poisson and logarithmic Poisson cohomology.

Recently in (Dongho, J. \& Yotcha, S. R., 2016), the Differential Point of view of such cohomology has been study and and apply in the prequantization of such Poisson manifold.

More general theory of logarithmic Poisson cohomology and logarithmic Poisson algebra is in preparation in (Dongho, J., et al.). 
The main results of this paper are:

Proposition 1 The Poisson cohomology groups of the Poisson algebra $\left(\mathcal{A}=C[x, y],\{x, y\}=x^{n}\right),\left(n \in N^{*}\right)$ are

$$
H_{P}^{0} \cong C, \quad H_{P}^{1} \cong C, \quad H_{P}^{k} \cong 0 \forall k \geq 2 \quad \text { when } \quad n=1
$$

and for all $n \geq 2$,

$$
\begin{aligned}
& H_{P}^{0}\left(x^{n}\right) \cong C \\
& H_{P}^{1}\left(x^{n}\right) \cong C_{n-1}[x] \oplus C[y] \oplus x C[y] \oplus \cdots \oplus x^{n-2} C[y] \\
& H_{P}^{2}\left(x^{n}\right) \cong C[y] \oplus x C[y] \oplus \cdots \oplus x^{n-2} C[y] \\
& H_{P}^{k}\left(x^{n}\right) \cong 0 \forall k \geq 3
\end{aligned}
$$

and

Proposition 2 The logarithmic Poisson cohomology groups of the Poisson structure defined by the logarithmic Poisson 2-form $\pi=x^{n} \partial x \wedge \partial y$, which is logarithmic along the ideal $I=x^{n} C[x, y]$ are

$$
\begin{aligned}
& H_{P S}^{n}\left(x^{n}\right) \simeq 0 \quad \text { for } \quad n \geq 3 \\
& H_{P S}^{2}\left(x^{n}\right) \simeq \underset{i=0}{\oplus-2} x^{i} C[y] \\
& H_{P S}^{1}\left(x^{n}\right) \simeq C_{n-1}[x] \oplus \underset{i=0}{\oplus-2} x^{i} C[y]
\end{aligned}
$$

Those can be generalize to the case of any algebra with two generator over a non zero characteristic ring.

\section{2. $A$-module of Differential Form}

It follows that, $A=C[x, y], \Omega_{A}$ is the $A$-module of differential form on $A$ and $D e r_{A}$ the $A$-module on derivations of $A$. Then, $\Omega_{A}=\langle d x, d y\rangle_{A}$ and $D e r_{A}=\langle\partial x, \partial y\rangle_{A}$.

For any $k \in N^{*}, A l t^{k}\left(\Omega_{A}, A\right)$ denote the $A$-module of $k$-multilinear skew symmetric form on $\Omega_{A}$. By convention, $A l t^{0}\left(\Omega_{A}, A\right)=A$ and then $A l t^{k}\left(\Omega_{A}, A\right) \cong \wedge^{k} \operatorname{Der}_{A}$. Then,

$$
\begin{aligned}
& A l t^{1}\left(\Omega_{A}, A\right) \cong D e r_{A} \cong A \times A \\
& A l t^{2}\left(\Omega_{A}, A\right) \cong \operatorname{Der}_{A} \wedge \operatorname{Der}_{A}=\langle\partial x \wedge \partial y\rangle_{A} \cong A \\
& A l t^{k}\left(\Omega_{A}, A\right) \cong 0 \text { pour tout } k \geq 3
\end{aligned}
$$

We deduce the following cochain complex

$$
0 \longrightarrow A \stackrel{d^{0}}{\longrightarrow} A \times A \stackrel{d^{1}}{\longrightarrow} A \stackrel{d^{2}}{\longrightarrow} 0
$$

where $d^{i}, i=0,1,2$ are associated Poisson differential and there are defined by

$$
\begin{aligned}
d^{0} \varphi(\alpha) & =H(\alpha) \varphi \text { for } \varphi \in A \\
d^{1} \varphi\left(\alpha_{1}, \alpha_{2}\right) & =H\left(\alpha_{1}\right) \varphi\left(\alpha_{2}\right)-H\left(\alpha_{2}\right) \varphi\left(\alpha_{1}\right)-\varphi\left(\left[\alpha_{1}, \alpha_{2}\right]\right) \text { for } \varphi \in A l t^{1}\left(\Omega_{A}, A\right), \alpha_{1}, \alpha_{2} \in \Omega_{A} \\
d^{k} & =0 \text { for every } k \geq 2
\end{aligned}
$$

$H: \Omega_{A} \longrightarrow D e r_{A}$ is the Hamiltonian map defined by $H(d a)=\{a,-\}$. It induce on $\Omega_{A}$ a bracket [,] defined by $[d a, d b]=d\{a, b\}$. In particular, $\left(\Omega_{A},[], H,\right)$ is a Lie-Rinehart-Poisson algebra. The cohomology groups are given by $H_{P}^{k}=\operatorname{ker} d^{k} / \operatorname{Im} d^{k-1}(k \in N)$. It therefore follows that

$$
H_{P}^{k} \cong 0 \quad \forall k \geq 3
$$

For a better understanding, we address the cases $n=1$ and $n=2$ and we end with a generalization.

\section{The Case $n=1$}

\subsection{Associated Poisson Differential}

Let $\varphi \in A l t^{0}\left(\Omega_{A}, A\right)=A, d^{0} \varphi \in A l t^{1}\left(\Omega_{A}, A\right) \cong \operatorname{Der}_{A}$ that is $d^{0} \varphi=\varphi_{1} \partial x+\varphi_{2} \partial y$ with $\varphi_{1}, \varphi_{2} \in A$. But $d^{0} \varphi(\alpha)=H(\alpha) \varphi$ for $\alpha \in \Omega_{A}$. Taking successively $\alpha=d x$ and $d y$ we obtain $\varphi_{1}=H(d x) \varphi$ and $\varphi_{2}=H(d y) \varphi$. 
On the other hand, $H(d x) \in \operatorname{Der}_{A}$ i.e; $H(d x)=\alpha_{1} \partial x+\alpha_{2} \partial y, \alpha_{1}, \alpha_{2} \in A$. So, $H(d x)(x)=\alpha_{1}=0$ and $H(d y)(x)=\alpha_{2}=$ $\{x, y\}=x$. Therefore $H(d x)=x \partial y$. Similarly we can show that $H(d y)=-x \partial x$ therefore

$$
d^{0} \varphi=x \frac{\partial \varphi}{\partial y} \partial x-x \frac{\partial \varphi}{\partial x} \partial y \approx\left(x \frac{\partial \varphi}{\partial y},-x \frac{\partial \varphi}{\partial x}\right)
$$

For $\varphi=\varphi_{1} \partial x+\varphi_{2} \partial y \in A l t^{1}\left(\Omega_{A}, A\right), d^{1} \varphi \in A l t^{2}\left(\Omega_{A}, A\right) \cong \wedge^{2} \operatorname{Der}_{A}$ i.e;

$d^{1} \varphi=\psi \partial x \wedge \partial y, \psi \in A$. Or $d^{1} \varphi\left(\alpha_{1}, \alpha_{2}\right)=H\left(\alpha_{1}\right) \varphi\left(\alpha_{2}\right)-H\left(\alpha_{2}\right) \varphi\left(\alpha_{1}\right)-\varphi\left(\left[\alpha_{1}, \alpha_{2}\right]\right)$ for $\varphi \in A l t^{1}\left(\Omega_{A}, A\right), \alpha_{1}, \alpha_{2} \in \Omega_{A}$. In particular, for $\alpha_{1}=d x$ and $\alpha_{2}=d y$, we get $\psi=H(d x) \varphi(d y)-H(d y) \varphi(d x)-\varphi([d x, d y])=x \frac{\partial \varphi_{2}}{\partial y}+x \frac{\partial \varphi_{1}}{\partial x}-\varphi_{1}$. Therefore,

$$
d^{1} \varphi=\left(x \frac{\partial \varphi_{1}}{\partial x}+x \frac{\partial \varphi_{2}}{\partial y}-\varphi_{1}\right) \partial x \wedge \partial y
$$

\subsection{Calculation of Cohomological Groups}

\subsubsection{Expression of $H_{P}^{0}(x)$}

By definition $H_{P}^{0}=\operatorname{ker} d^{0} / \operatorname{Im} d^{-1}$ with $d^{-1}: 0 \longrightarrow A$ i.e; $H^{0}=\operatorname{ker} d^{0}$. Let $\varphi \in A, d^{0} \varphi=0$ If and only if $x \frac{\partial \varphi}{\partial x}=x \frac{\partial \varphi}{\partial y}=0$ That is $\varphi \in C$. Therefore,

$$
H_{P}^{0} \cong C
$$

3.2.2 Expression of $H_{P}^{2}=\operatorname{ker} d^{2} / \operatorname{Im} d^{1}$

Obviously, $\operatorname{ker} d^{2}=A$ Let $\varphi \in A$, we can write

$$
\varphi=-(-\varphi)+x \frac{\partial(-\varphi)}{\partial x}+x \frac{\partial}{\partial y}\left(\int \frac{\partial \varphi}{\partial x} d y\right)=d^{1}\left(-\varphi, \int \frac{\partial \varphi}{\partial x} d y\right)
$$

$d^{1}$ is an epimorphism and we have

$$
H_{P}^{2} \cong 0
$$

\subsubsection{Expression of $H_{P}^{1}(x)$}

$\left(\varphi_{1}, \varphi_{2}\right) \in \operatorname{ker} d^{1}$ if and only if $\varphi_{1}=x\left(\frac{\partial \varphi_{2}}{\partial y}+\frac{\partial \varphi_{1}}{\partial x}\right)$. Thus, we can write $\varphi_{1}=x u, u \in A$ therefore $\varphi_{2}=-x \int \frac{\partial u}{\partial x} d y+b(x)$ with $b(x) \in C[x]$. It was therefore

$$
\operatorname{ker} d^{1}=\left\{\left(x u,-x \int \frac{\partial u}{\partial x} d y\right)+(0, b(x)) ; u \in A, b(x) \in C[x]\right\}
$$

Let

$$
\begin{aligned}
\beta: A & \longrightarrow x A \times A \\
u & \longmapsto\left(x u,-x \int \frac{\partial u}{\partial x} d y\right)
\end{aligned}
$$

$\beta$ is a monomorphism and we have

$$
\operatorname{ker} d^{1}=\beta(A) \oplus(0 \times C[x])=\beta(A) \oplus(0 \times x C[x]) \oplus(0 \times C)
$$

in other hand, $\beta(A) \oplus(0 \times x C[x]) \subseteq d^{0}(A)$ and $d^{0}(A) \cap(0 \times C)=0$. Therefore, ker $d^{1}=\beta(A) \oplus(0 \times x C[x]) \oplus(0 \times C) \subseteq$ $d^{0}(A) \oplus(0 \times C) \subseteq \operatorname{ker} d^{1}$. We deduce that $\operatorname{ker} d^{1}=d^{0}(A) \oplus(0 \times C)$; that is

$$
H_{P}^{1} \cong C
$$

\section{The Case $n=2$}

In this section, we recall and generalize the methods and results obtained in (Dongho, J., 2012). By using the reasoning above, the associated Poisson differential are:

$$
d^{0} \varphi=x^{2} \frac{\partial \varphi}{\partial y} \frac{\partial}{\partial x}-x^{2} \frac{\partial \varphi}{\partial x} \frac{\partial}{\partial y}, \quad \varphi \in A
$$

and

$$
d^{1}\left(\varphi_{1}, \varphi_{2}\right)=x^{2} \frac{\partial \varphi_{1}}{\partial x}+x^{2} \frac{\partial \varphi_{2}}{\partial y}-2 x \varphi_{1}, \quad \varphi_{1}, \varphi_{2} \in A
$$




\subsection{Explicit Expression of Associated Cohomological Groups}

\subsubsection{Expression of $H_{P}^{0}\left(x^{2}\right)$ and $H_{P}^{1}\left(x^{2}\right)$}

By direct computation, we have:

$$
H_{P}^{0} \cong C
$$

By definition, we have $\operatorname{ker} d^{2}=A$ and $\operatorname{Im} d^{1} \subseteq x A$. For $\varphi \in A$, we have

$$
x \varphi=-2 x\left(-\frac{1}{2} \varphi\right)+x^{2} \partial x\left(-\frac{1}{2} \varphi\right)+x^{2} \partial y\left(\int \partial x\left(\frac{1}{2} \varphi\right) d y\right)=d^{1}\left(-\frac{1}{2} \varphi, \int \partial x\left(\frac{1}{2} \varphi\right) d y\right) .
$$

That is $\operatorname{Im} d^{1}=x A$. In other hand $A=C[y] \oplus x A$. Therefore

$$
H_{P}^{2}\left(x^{2}\right) \cong C[y]
$$

\subsubsection{Expression of $H_{P}^{1}\left(x^{2}\right)$}

Let $\varphi_{1}, \varphi_{2} \in A ;\left(\varphi_{1}, \varphi_{2}\right)$ is a 1-cocycle if and only if $2 \varphi_{1}=x\left(\frac{\partial \varphi_{1}}{\partial x}+\frac{\partial \varphi_{2}}{\partial y}\right)$. We deduce that $\varphi_{1}=x u, u \in A$ and consequently $\varphi_{2}=\int(1-x \partial x) u d y+b(x)$ with $b(x) \in C[x]$ i.e;

$$
\operatorname{ker} d^{1}=\left\{\left(x u, \int(1-x \partial x) u d y\right)+(0, b(x)), u \in A, b(x) \in C[x]\right\}
$$

Let

$$
\begin{aligned}
\beta: A & \longrightarrow x A \times A \\
u & \longmapsto\left(x u, \int(1-x \partial x) u d y\right)
\end{aligned}
$$

$\beta$ is a monomorphism and we have $\operatorname{ker} d^{1}=\beta(A) \oplus(0 \times C[x])$. Since $A=C[y] \oplus x A$, we obtain

$$
\operatorname{ker} d^{1}=\beta(C[y]) \oplus \beta(x A) \oplus\left(0 \times x^{2} C[x]\right) \oplus\left(0 \times C_{1}[x]\right)
$$

where $C_{1}[x]=\left\{a_{0}+a_{1} x ; a_{0}, a_{1} \in C\right\}$ is the vector space of polynomials of degree less than or equal to 1 . In other hand, we have $\beta(x A) \oplus\left(0 \times x^{2} C[x]\right) \subseteq d^{0}(A)$ et $d^{0}(A) \cap\left[\left(0 \times C_{1}[x]\right) \oplus \beta(C[y])\right]=0$. Therefore

$$
\operatorname{ker} d^{1} \subseteq d^{0}(A) \oplus \beta(C[y]) \oplus\left(0 \times C_{1}[x]\right) \subseteq \operatorname{ker} d^{1}
$$

i.e;

$$
\operatorname{ker} d^{1}=d^{0}(A) \oplus \beta(C[y]) \oplus\left(0 \times C_{1}[x]\right)
$$

then

$$
H_{P}^{1} \cong C_{1}[x] \oplus C[y]
$$

\section{Generalization $(n \geq 2)$}

At this stage, the calculation of differentials is no longer a secret. We therefore obtains

$$
\begin{aligned}
d^{0} \varphi & =\left(x^{n} \frac{\partial \varphi}{\partial y},-x^{n} \frac{\partial \varphi}{\partial x}\right) \\
d^{1}\left(\varphi_{1}, \varphi_{2}\right) & =x^{n} \frac{\partial \varphi_{1}}{\partial x}+x^{n} \frac{\partial \varphi_{2}}{\partial y}-n x^{n-1} \varphi_{1}
\end{aligned}
$$

with $\varphi, \varphi_{1}, \varphi_{2} \in A$.

\subsection{Calculation of Associated Poisson Cohomological Groups}

In this section, we compute all Poisson cohomological groups associated to the above Poisson complex.

5.1.1 Calculation of $H_{P}^{0}$

For any $\varphi \in A$, we have $d^{0} \varphi=0$ if and only if $\varphi=c t e \in C$. We deduce that

$$
H_{P}^{0} \cong C
$$




\subsubsection{Calculation of $H_{P}^{2}$}

By definition, $\operatorname{ker} d^{2}=A$ and $\operatorname{Im} d^{1} \subseteq x^{n-1} A$. For $u \in A$, we have

$$
x^{n-1} u=-n x^{n-1}\left(-\frac{1}{n} u\right)+x^{n} \frac{\partial}{\partial x}\left(-\frac{1}{n} u\right)+x^{n} \frac{\partial}{\partial y}\left(\int \frac{\partial}{\partial x}\left(\frac{1}{n} u\right) d y\right)
$$

We deduce that $\operatorname{Im} d^{1}=x^{n-1} A$. In addition,

$$
A=C[y] \oplus x C[y] \oplus \cdots \oplus x^{n-2} C[y] \oplus x^{n-1} A .
$$

Therefore

$$
H_{P}^{2} \cong C[y] \oplus x C[y] \oplus \cdots \oplus x^{n-2} C[y]
$$

\subsubsection{Calculation of $H_{P}^{1}$}

Let $\left(\varphi_{1}, \varphi_{2}\right) \in A^{2}, d^{1}\left(\varphi_{1}, \varphi_{2}\right)=0$ if and only if $n \varphi_{1}=x\left(\frac{\partial \varphi_{1}}{\partial x}+\frac{\partial \varphi_{2}}{\partial y}\right)$. Thus $\varphi_{1}=x u$ with $u \in A$ and consequently $\varphi_{2}=\int\left(n-1-x \frac{\partial}{\partial x}\right) u d y+b(x)$ with $b(x) \in C[x]$. Therefore,

$$
\operatorname{ker} d^{1}=\left\{\left(x u, \int\left(n-1-x \frac{\partial}{\partial x}\right) u d y\right)+(0, b(x)), u \in A, b(x) \in C[x]\right\}
$$

Consider the application

$$
\begin{aligned}
\beta: A & \longrightarrow x A \times A \\
u & \longmapsto\left(x u, \int\left(n-1-x \frac{\partial}{\partial x}\right) u d y\right)
\end{aligned}
$$

$\beta$ is a monomorphism and we have ker $d^{1}=\beta(A) \oplus(0 \times C[x])$. On the other hand, $A=C[y] \oplus x C[y] \oplus \cdots \oplus x^{n-2} C[y] \oplus x^{n-1} A$. Therefore

$$
\operatorname{ker} d^{1}=\beta(C[y]) \oplus \beta(x C[y]) \oplus \cdots \oplus \beta\left(x^{n-2} C[y]\right) \oplus \beta\left(x^{n-1} A\right) \oplus\left(0 \times x^{n} C[x]\right) \oplus\left(0 \times C_{n-1}[x]\right)
$$

with $C_{n-1}[x]=\left\{a_{0}+a_{1} x+a_{2} x^{2}+\cdots+a_{n-1} x^{n-1} ; a_{0}, \cdots, a_{n-1} \in C\right\}$ denoting the vector space of polynomials of degree less than $n-1$. Let us show now that $\beta\left(x^{n-1} A\right) \oplus\left(0 \times x^{n} C[x]\right) \subseteq d^{0}(A)$. Let $u \in A$ and $b(x) \in C[x]$,

$$
\begin{aligned}
\beta\left(x^{n-1} u\right)+\left(0, x^{n} b(x)\right) & =\left(x^{n} u, \int\left(n-1-x \frac{\partial}{\partial x}\right)\left(x^{n-1} u\right) d y+x^{n} b(x)\right) \\
& =\left(x^{n} u,-x^{n} \int \frac{\partial u}{\partial x} d y+x^{n} b(x)\right) \\
& =\left(x^{n} u,-x^{n} \frac{\partial}{\partial x}\left[\iint \frac{\partial u}{\partial x} d y d x-\int b(x) d x\right]\right) \\
& =\left(x^{n} \frac{\partial}{\partial y}\left[\int u d y-\int b(x) d x\right],-x^{n} \frac{\partial}{\partial x}\left[\int u d y-\int b(x) d x\right]\right) \\
& =d^{0}\left(\int u d y-\int b(x) d x\right)
\end{aligned}
$$

By a simple computation, we have

$$
d^{0}(A) \cap\left(\beta(C[y]) \oplus \beta(x C[y]) \oplus \cdots \oplus \beta\left(x^{n-2} C[y]\right) \oplus\left(0 \times C_{n-1}[x]\right)\right)=0
$$

Furthermore, $\beta(C[y]), \beta(x C[y]), \cdots, \beta\left(x^{n-2} C[y]\right),\left(0 \times C_{n-1}[x]\right), d^{0}(A)$ are parts of ker $d^{1}$. We deduce that

$$
\operatorname{ker} d^{1} \subseteq d^{0}(A) \oplus \beta(C[y]) \oplus \beta(x C[y]) \oplus \cdots \oplus \beta\left(x^{n-2} C[y]\right) \oplus\left(0 \times C_{n-1}[x]\right) \subseteq \operatorname{ker} d^{1}
$$

and then,

$$
\operatorname{ker} d^{1}=d^{0}(A) \oplus \beta(C[y]) \oplus \beta(x C[y]) \oplus \cdots \oplus \beta\left(x^{n-2} C[y]\right) \oplus\left(0 \times C_{n-1}[x]\right)
$$

since $\beta$ is a monomorphism, we have

$$
H_{P}^{1} \cong C_{n-1}[x] \oplus C[y] \oplus x C[y] \oplus \cdots \oplus x^{n-2} C[y]
$$


Proposition 3 The Poisson cohomology groups of the Poisson algebra $\left(\mathcal{A}=C[x, y],\{x, y\}=x^{n}\right),\left(n \in N^{*}\right)$ are

$$
H_{P}^{0} \cong C, \quad H_{P}^{1} \cong C, \quad H_{P}^{k} \cong 0 \forall k \geq 2 \quad \text { when } \quad n=1
$$

and for all $n \geq 2$,

$$
\begin{aligned}
& H_{P}^{0} \cong C \\
& H_{P}^{1} \cong C_{n-1}[x] \oplus C[y] \oplus x C[y] \oplus \cdots \oplus x^{n-2} C[y] \\
& H_{P}^{2} \cong C[y] \oplus x C[y] \oplus \cdots \oplus x^{n-2} C[y] \\
& H_{P}^{k} \cong 0 \forall k \geq 3
\end{aligned}
$$

\section{Associated Logarithmic Poisson Cohomology}

The Poisson 2-form remain $\pi=x^{n} \partial_{x} \wedge \partial_{y}$ and the module of 1-form logarithmic along $x^{n} A$ is $\Omega_{A}(\log I)=\frac{d x}{x} C[x, y] \oplus$ $C[x, y] d y$ and the associated logarithmic Hamiltonian map is $H\left(\frac{d x}{x}\right)=x^{n-1} \partial_{y}, H(d y)=-x^{n} \partial_{x}$. This Hamiltonian map induced the following complex $0 \longrightarrow A \stackrel{\partial^{0}}{\longrightarrow} A \otimes A \stackrel{\partial^{1}}{\longrightarrow} A \longrightarrow 0$. Where $\partial^{0} f=x^{n-1}(\partial y f,-x \partial x f)$ and $\partial^{1}\left(f_{1}, f_{2}\right)=$ $x^{n-1}\left(\partial y f_{2}+x \partial x f_{1}-(n-1) f_{1}\right)$. It follow that The order zero logarithmic Poisson cohomology group is $H_{P S}^{0} \simeq C$. In order to determine $H_{P S}^{1}$ and $H_{P S}^{2}, A$ is decomposed as follows: $A=C[y] \oplus x C[y] \oplus \ldots \oplus x^{n-2} \oplus x^{n-1} C[x, y]$. So; for all $g_{0}(y)+x g_{1}(y)+\ldots+x^{n-2} g(y)+x^{n-1} g_{n-1}(x, y)=g(x, y) \in A$, we have, $g \in \partial^{1}(A)$ if and only if $g_{n-1}(y)=\partial y f_{2}+x \partial x f_{1}-f_{1}$ and $g_{i}=0$ for all $i \in\{0, \ldots, n-2, n\}$; for some $f_{1}, f_{2} \in A$. Therefore, given $f_{1} \in A$, there exist $a(x) \in C[x]$ such that $f_{2}=\int\left(g_{n-1}+(n-1) f_{1}-x \partial x f_{1}\right) d y+a(x)$. In particular, for $f_{1}=g_{n-1}$ and $a(x)=0$, we have $f_{2}=\int\left(n g_{n-1}-x \partial x g_{n-1}\right) d y$. Moreover, for all $0 \neq g_{0}(y)+x g_{1}(y)+\ldots+x^{n-2} g(y)$, the following equation

$$
x^{n-1}\left(\partial y f_{2}+x \partial x f_{1}-(n-1) f_{1}\right)=g_{0}(y)+x g_{1}(y)+\ldots+x^{n-2} g(y) .
$$

haven't solution in $A \otimes A$. This implies that $A \simeq \partial^{1}(A \otimes A) \oplus \underset{i=0}{\oplus} x^{i} C[y]$. Therefore

$$
H_{P S}^{2} \simeq \bigoplus_{i=0}^{n-2} x^{i} C[y]
$$

Let $\left(f_{1}, f_{2}\right) \in A \otimes A$. It is an element of $\operatorname{Ker}\left(\partial^{1}\right)$ if and only if $\partial y f_{2}=(n-1) f_{1}-x \partial x f_{1}$. That is $f_{2}=\int\left((n-1) f_{1}-\right.$ $\left.x \partial x f_{1}\right) d y+b(x)$. We define the following map $A \stackrel{\eta}{\longrightarrow} A \otimes A$ by $\eta(u)=\left(u, \int((n-1) u-x \partial x u) d y\right)$. It is a monomorphism of $C$-modules and it follows from the above description of $K e r \partial^{1}$ that

$$
\begin{aligned}
K e r \partial^{1} & \simeq \eta(A) \oplus C[x] \\
& \simeq \eta\left({ }_{i=0}^{n-2} x^{i} C[y]\right) \oplus \eta\left(x^{n-1} C[x, y]\right) \oplus x^{n} C[x] \oplus C_{n-1}[x]
\end{aligned}
$$

In addition, for all $g \in \eta\left(x^{n-1} C[x, y] \oplus\left(O_{A} \times x^{n} C[x]\right)\right)$, there exist $u \in C[x, y]$ and $v \in C[x]$ such that $g=\eta\left(x^{n-1} u\right)+$ $\left(0, x^{n} v(x)\right)=x^{n-1}\left(u,-x\left(\int \partial y u d y-v\right)\right)$. This element is in $\partial^{0}(A)$ if and only if, there exist $a \in A$ such that $\partial^{0}(a)=g$. This imply that there exist $c(x) \in C[x]$ such that $a=\int u d y+c(x)$ and $\partial x(a)=\partial x u d y-v(x)$. But this imply that $c(x)=-\int v(x) d x$ and then $a=\int u d y-\int v(x) d x$ and $\eta\left(x^{n-1} C[x, y] \oplus\left(O_{A} \times x^{n} C[x]\right)\right) \subset \partial^{0}(A)$. In other hand the following equation in $u$ have no solution in $C[x, y]$

$$
\left\{\begin{aligned}
x^{n-1} \partial y u & =\sum_{i=0}^{n-2} x^{i} g_{i}(y) \\
x^{n} \partial x u= & \sum_{i=0}^{n-2} x^{i} \int g_{i}(y) d y+\sum_{i=0}^{n-1} a_{i} x^{i}
\end{aligned}\right.
$$

Therefore $K \operatorname{er} \partial^{1} \simeq C_{n-1}[x] \oplus \eta\left(\underset{i=0}{n-2} x^{i} C[y]\right) \oplus \partial^{0}(A)$ and then

$$
H_{P S}^{1}\left(x^{n}\right) \simeq C_{n-1}[x] \oplus \eta\left(\oplus_{i=0}^{n-2} x^{i} C[y]\right)
$$


This complete the proof of the following proposition

Proposition 3 The logarithmic Poisson cohomology groups of the Poisson structure defined by the logarithmic Poisson 2-form $\pi=x^{n} \partial x \wedge \partial y$, which is logarithmic along the ideal $I=x^{n} C[x, y]$ are

$$
\begin{aligned}
& H_{P S}^{n} \simeq 0 \quad \text { for } \quad n \geq 0 \\
& H_{P S}^{2} \simeq \bigoplus_{i=0}^{n-2} x^{i} C[y] \\
& H_{P S}^{1}\left(x^{n}\right) \simeq C_{n-1}[x] \oplus \eta\left(\oplus_{i=0}^{n-2} x^{i} C[y]\right)
\end{aligned}
$$

\section{Acknowledgement}

We will like to thanks Mister Shuntah Roland Yotcha that accepted to carry out the necessary English translation.

\section{References}

Braconnier, J. (1977). Algèbres de Poisson. C. R. Acad. Sci. Paris Sér, A-B 284(21). A1345-A1348.

Deligne, P. Equations Diffrentielles à Points Singuliers Réguliers. Lecture Notes in Mathematics. Berlin. Heidelberg. New York.

Dongho, J. (2012). Logarithmic Poisson cohomology: example of calculation and application to prequantization. Annales de la facult?des sciences de Toulouse Mathématiques, 21(4), 623-650. https://doi.org/10.5802/afst.1347

Dongho, J., \& Yotcha, S. R. (2016). On logarithmic Prequantization of logarithmic Poisson manifolds. Journal of Mathematical Sciences and Applications, 4(1), 4-13. http://dx.doi.org/10.12691/jmsa-4-1-2

Dongho, J., Yotcha, S. R., \& Mbah, A. On Theory of logarithmic Poisson Cohomology in preparation.

Goto, R. (2002). Rozansky-Witten Invariants of log symplectic Manifolds. Contemporary Mathematics, 309, 69-84. https://doi.org/10.1090/conm/309/05342

Hochschild, G., Kostant, B., \& Rosenberg, A. (1962). Differential Forms On Regular Affine Algebras. Trans. Amer. Math. Soc., 102, 383-408. https://doi.org/10.1090/S0002-9947-1962-0142598-8

Huebschmann, J. (1990). Poisson Cohomology and quantization, J.Reine Angew. Math., 408, 57-113.

Krasil'shchik, I. (1980). Hamiltonian cohomology of canonical algebras. Dokl. Akad. Nauk SSSR, 251(6), 1306-1309.

Lie, S. (1890). Theorie der Transformations gruppen, (Zweiter Abschnitt, unter Mitwirkung von Prof. Dr. Friederich Engel), Teubner, Leipzig.

Palais, R. (1961). The cohomology of Lie ring. Proc. Symp. Pure Math., 3, 130-137. https://doi.org/10.1090/pspum/003/0125867

Pichereau, A. (2006). Poisson (co)homology and isolated singularities. J. Algebra, 299(2), 747-777. https://doi.org/10.1016/j.jalgebra.2005.10.029

Rinehart, G. (1963). Differential forms on general commutative algebras. Trans. Amer. Math. Soc., 108(2), 195-222. https://doi.org/10.1090/S0002-9947-1963-0154906-3

Polishchuk, A. (1997). Algebraic geometry of poisson brackets, Journal of Mathematical Sciences., 84(5).

Monnier, Ph. (2002). Poisson cohomology in dimension two. Israel J. Math., 129, 189-207.

Saito, K. (1980). Theory of logarithmic differential forms and logarithmic vector fields, Sec. IA, J.Fac.Sci. Univ. Tokyo., 27, 265-291.

Vaisman, I. (1991). On the geometric quantization of Poisson manifolds. J. Math., 32, 3339-3345. https://doi.org/10.1063/1.529446

Vinogradov, A. M., \& Krasil'shchik, I. S. (1975). What is Hamiltonian formalism? Russian, Uspehi Mat. Nauk, 30(1), 173-198.

Weinstein, A. (1983). The local structure of Poisson manifolds. J. Differential Geometry, 18, 523-557.

\section{Copyrights}

Copyright for this article is retained by the author(s), with first publication rights granted to the journal. 
This is an open-access article distributed under the terms and conditions of the Creative Commons Attribution license (http://creativecommons.org/licenses/by/4.0/). 